\title{
LA IMPOTENCIA DE LAS REPRESENTACIONES: UNA crítica a la ontología social de John Searle
}

THE IMPOTENCE OF REPRESENTATIONS: A CRITICISM OF JOHN SEARLE'S SOCIAL ONTOLOGY

\author{
José GIROMINI* \\ Universidad Nacional de Córdoba, Córdoba, Argentina. jgiromini@gmail.com
}

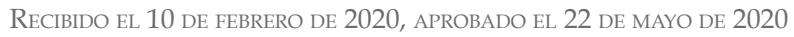

\begin{abstract}
Resumen
John Searle ha defendido un enfoque intencionalista de lo social, a saber, la idea de que la realidad social es creada y mantenida por ciertos estados mentales y actos de habla. En este trabajo, en primer lugar, presentamos una versión detallada de los argumentos fundacionales de Searle, basada en la conexión entre reconocimiento colectivo y lenguaje. En segundo lugar, ofrecemos una crítica de estos argumentos, sugiriendo que ellos mismos nos llevan a admitir que los fundamentos de la realidad social son más prácticos que representacionales.
\end{abstract}

\section{Palabras clave}

Ontología social, normatividad, John Searle, filosofía de la mente, pragmatismo.

\section{Abstract}

John Searle has advocated an intentionalist approach to the social, namely the idea that social reality is created and maintained by certain mental states and speech acts. In this work, we first present a detailed version of Searle's intentionalist founding arguments based on the connection between collective recognition and language. In the second place, we offer a critique of these arguments, suggesting that they lead us to admit that the foundations of social reality are more practical than representational.

\section{KEY WORDS}

Social ontology, normativity, John Searle, philosophy of mind, pragmatism.

* (D) orcid.org/0000-0003-3893-6843 Google Scholar 


\section{Introducción}

De la ontología social de John Searle puede decirse que es una derivación, casi una consecuencia secundaria, de un sistema filosófico organizado alrededor de la explicación de la mente y el lenguaje. Aunque el autor haya dedicado sus dos libros más recientes, Making the Social World (2010) y Money, Social Ontology and Law (2019), a elaborar y difundir este aspecto de su trabajo, es sabido que el grueso de su producción filosófica ha girado en torno a temas como la naturaleza de la intencionalidad, la percepción, la estructura de los estados mentales, la acción racional y, por supuesto, la teoría de los actos de habla. No es que Searle haya puesto más curiosidad en los fenómenos intencionales y menos en la ontología social: el carácter derivativo de la segunda respecto a los primeros obedece a razones conceptuales. La ontología social de Searle es la aplicación de una teoría de la mente y el lenguaje para la explicación la sociedad. Existe en su filosofía un orden lógico muy claro, y permanentemente enfatizado, que sostiene que la realidad social es el producto de ciertos funcionamientos lingüísticos y mentales. Queremos en este trabajo cuestionar esta filosofía intencionalista de lo social, oponiéndole una concepción pragmatista, que enfatiza la importancia de la acción para la creación de la realidad social.

Aunque Searle se haya ocupado, especialmente en su último volumen, de elucidar la posibilidad y el funcionamiento de entidades sociales concretas como el dinero, el poder político, las corporaciones o los derechos humanos, aquí nos ocuparemos de sus esfuerzos fundacionales, presentados originalmente en The Construction of Social Reality (1994) y elaborados ulteriormente en Making the Social World (2010), trabajo al que dedicaremos la mayor parte de nuestra atención. Cuando hablamos de esfuerzos fundacionales, nos referimos a que Searle se enorgullece de haber dado respuesta a un profundo problema filosófico: cómo es posible la sociedad. Y no sólo de eso, sino también de haber dado una respuesta compatible con un proyecto naturalista de largo alcance donde se explica, en palabras del autor, "cómo es posible que en un universo que consiste enteramente en partículas físicas dentro de campos de fuerza existan cosas tales como la consciencia, el libre albedrío, la sociedad y las obligaciones" (Searle, Making 3).

El orden del trabajo es el siguiente: en I, presentaremos la concepción general de la realidad social que defiende Searle y los elementos básicos de la explicación que ofrece de ella. En II, consideraremos con detalle 
los argumentos que Searle avanza para ofrecer su fundamentación intencionalista de la sociedad, basándonos en una interpretación del vínculo conceptual que existe entre reconocimiento colectivo y lenguaje. En III, nos dedicaremos a cuestionar estos argumentos, mostrando que, a partir de sus propias insuficiencias, es posible mostrar que la sociedad tiene una fundamentación más pragmática que representacional. En IV, con el objetivo de subrayar su especificidad, comparamos someramente nuestras objeciones con otras que se han presentado contra el programa de Searle.

\section{La descripción y la explicación del mundo social}

\section{Tres aspectos de la realidad social}

Podemos comenzar nuestra exposición de la ontología social de Searle señalando la que éste considera como la diferencia específica de la realidad social. Esta diferencia puede ser presentada bajo varios aspectos. El autor nos habla a veces de que la especificidad de las sociedades humanas se cifra en que podemos imponer funciones -que denomina funciones de estatus- a objetos y personas (Searle, Making 7), funciones que estos no podrían cumplir solamente en virtud de su estructura física, otras insiste en que la realidad social es un estrato compuesto por hechos institucionales (Searle, Making 10), finalmente, define los fenómenos sociales como poseyendo poderes deónticos que van más allá de la semántica (Searle, Making 9).

\section{Hechos institucionales}

Tomemos como hilo conductor el más ontológico de estos modos de capturar lo social. La realidad social está compuesta por lo que Searle denomina hechos institucionales -y que quizás sea más esclarecedor llamar hechos instituidos- en oposición a los hechos brutos. Resulta en principio sencillo ubicar hechos de uno u otro lado. Que Plutón se encuentre en este momento a una distancia determinada de Urano es un hecho bruto, que algunas personas discutan conjeturas astronómicas y astrológicas basándose en esa distancia es un hecho institucional. Que exista una gran reserva de petróleo en alguna profundidad terrestre es un hecho bruto, que su descubrimiento acarree un descenso del precio del petróleo es un hecho institucional. De los hechos institucionales, Searle (Making 10) nos dice un poco escuetamente que requieren de instituciones para existir; siendo más generoso, agrega que una institución es un sistema de reglas constitutivas y que la existencia de dicho sistema automáticamente crea la posibilidad de los hechos institucionales. 
La realidad social es, pues, en primer lugar, una realidad institucional. Y las instituciones deben entenderse como sistemas de reglas constitutivas. Aquí aparece un nuevo contraste, muy utilizado en la filosofía del lenguaje y del derecho, entre reglas regulativas y reglas constitutivas. Las primeras se encargan de regular, en el sentido de dotar de significación normativa, hechos que pueden ser especificados o que existen independientemente de la regla. El acto de comer no precisa de los consejos de Erasmo de Rotterdam para ser inteligible; ni el acto de manejar, en principio, de la educación vial. Las reglas constitutivas, por el contrario, crean la posibilidad de los comportamientos que regulan (Searle, Making 10): los actos que gobiernan no son especificables sin presuponer esas reglas. Ninguna descripción del orden de ciertas piezas esculpidas como caballos, reyes o torres alcanza para que podamos especificarla como un jaque si no se asumen las reglas del ajedrez. Las reglas constitutivas son, pues, condiciones de la existencia de ciertas situaciones -jaque, elecciones presidenciales-, ciertos actos -enroque, votar a tal candidato- e incluso de ciertas entidades -rey, presidente ${ }^{1}$.

El esquema de las reglas constitutivas, de acuerdo con Searle, es el siguiente: " $X$ cuenta como $Y$ en $C$ "; donde " $X$ " se especifica en términos externos a la regla, " $Y$ " en términos internos y " $C$ " hace referencia más o menos directa al sistema de reglas. Una papeleta de tales o cuales características $(\mathrm{X})$ cuenta como un voto $(\mathrm{Y})$ en el marco de las elecciones presidenciales (C). Que " $X$ " se especifique en términos externos a la regla constitutiva en cuestión no implica que deba ser especificada en términos externos a toda regla constitutiva, así: Un ciudadano argentino (X) cuenta como el presidente (Y) sí obtuvo la mayoría de los votos en las elecciones $(\mathrm{C})$.

\section{Funciones de estatus}

Ahora bien, ¿en qué consiste que a un objeto o persona $X$ se le asigne, mediante una regla constitutiva, el estatus social $Y$ en ciertas condiciones? Consiste, de acuerdo con Searle, en un cambio en sus propiedades funcionales. Dicho objeto o persona se encuentra habilitado, en virtud del estatus que ahora detenta, a cumplir ciertos roles que antes no estaban disponibles. Una persona dotada del estatus de secretario general de un gremio puede llamar a una huelga y un dibujo que cuente como la firma de alguien puede consumar la obligación de pagar una deuda.

\footnotetext{
${ }^{1}$ Para una crítica de que esta distinción pueda trazarse con claridad en diferentes casos sociales relevantes -como la guerra, por ejemplo- (Sánchez Cuenca, “A Behavioral”).
} 
Las funciones que dependen de reglas constitutivas para existir llevan el nombre de funciones de estatus. Searle las define, cabe recordar, como funciones que objetos y personas consiguen desempeñar pero que no podrían desempeñar sólo en virtud de su estructura física. Un martillo puede, en virtud de su estructura física, desempeñar la función de asegurar un clavo, pero solo en virtud de encontrarse involucrado en una serie de reglas de procedimientos penales, puede contar como evidencia. Más aún, las funciones de estatus con las que los objetos y las personas son socialmente investidos no necesitan ser impuestas sobre objetos físicos "pelados", es decir, ausentes de otras determinaciones sociales. De hecho, lo contrario suele ser muy frecuente. Por este motivo, podemos definir más precisamente las funciones de estatus como aquellas que un objeto o una persona no podrían cumplir si no fuera porque se encuentran dentro de un sistema específico de reglas constitutivas ${ }^{2}$.

\section{Poderes deónticos}

Resta agregar un elemento más, correspondiente al tercer aspecto bajo el cual Searle presenta la especificidad de lo social: los poderes deónticos. Podemos introducirlo apelando a una dicotomía tradicional: si no es en virtud de sus propiedades físicas o poderes causales que los objetos o personas consiguen desempeñar las funciones de estatus, ¿en virtud de qué pueden hacerlo? A través de lo que Searle llama sus poderes deónticos. Estar investido de un determinado estatus -sea el de dinero, presidente o evidencia- consiste no en otra cosa que en encontrarse involucrado en una red de permisos, obligaciones, derechos, autorizaciones, etc. (Searle, Making 8). Para un objeto X, contar como dinero (Y) consiste simplemente en que sus poseedores están autorizados a utilizarlo como medio de intercambio, ahorro, pago, etc. Para una persona $X$, contar como estudiante universitario $(\mathrm{Y})$ consiste en estar autorizado a inscribirse en ciertos cursos, estar en muchos países obligado a pagar cierto arancel, encontrarse sujeto a determinado código de conducta, etc. Como principio general: la expresión "cuenta como" de las reglas constitutivas siempre se especifica en términos deónticos o normativos.

\footnotetext{
${ }^{2}$ Un asunto que ha suscitado bastante debate alrededor de las funciones de estatus, introducido por Barry Smith ("John Searle"), es el de los free-standing Y terms, que podríamos traducir como términos $Y$ autónomos. Se trata de casos en que no existe claramente un objeto físico que es investido de un estatus $Y$; las $Y$ sociales comienzan a existir, aparentemente, sin una realización física. El caso más ejemplar es el de las personas jurídicas como las corporaciones. No creemos que se trate de una objeción mayor contra el esquema de Searle. Su respuesta se encuentra en Searle (Making 20).
} 
Ensayemos, pues, la siguiente somera descripción: la realidad social consiste en un agregado de hechos institucionales que derivan de reglas constitutivas. Estas reglas, a su vez, invisten a objetos y personas de ciertos estatus, y la posesión de esos estatus se entiende en términos de configuraciones de poderes deónticos. Resta entonces explicar cómo ese conjunto de entidades -instituciones, reglas, estatus y poderes deónticosson posibles.

\section{La explicación intencionalista del mundo social}

El dominio de lo social surge cuando conseguimos sobreañadir, a través de reglas constitutivas, un estrato de hechos institucionales encima de un estrato de hechos brutos. En este punto, Searle comparte los parámetros naturalistas de la mayor parte de la filosofía contemporánea. La especificidad de su teoría reside, en cambio, en la explicación del surgimiento y operación, de la institución y la influencia, de este estrato de hechos institucionales.

Podríamos catalogar la teoría de Searle como un enfoque intencionalista, pues los mecanismos de institución y funcionamiento de la realidad social que propone son, principalmente, dos fenómenos intencionales: por un lado, el reconocimiento colectivo -a saber, ciertos estados mentales muy especiales- $\mathrm{y}$, por otro, las declaraciones -a saber, ciertos actos de habla muy especiales.

\section{Reconocimiento colectivo}

El reconocimiento colectivo pertenece a esa categoría de estados mentales que la reciente filosofía analítica ha dado en llamar intencionalidad colectiv $a^{3}$ y que se caracterizan, precisamente, porque de ellos puede decirse que son tenidos por grupos.

La cuestión de la intencionalidad colectiva ha suscitado numerosos debates. El principal de ellos ha girado alrededor de elucidar si hablar de intenciones, creencias o acciones colectivas constituye la atribución directa de estados mentales a colectivos de personas o si se trata de una forma abreviada de referir a la distribución de estados mentales individuales entre sus miembros ${ }^{4}$. En relación a este punto, Searle ofrece, en la misma página, dos definiciones del reconocimiento colectivo que,

\footnotetext{
${ }^{3}$ Para una introducción a este debate, puede consultarse la compilación de Chant, Hindriks y Preyer (From individual).

${ }^{4}$ Estas dos opciones corresponden, en términos generales, a los enfoques ya clásicos de Margaret Gilbert (Joint Committment) y Bratman ("Shared Intention”).
} 
si bien se alinean con la segunda opción, se diferencian en sus grados de exigencia. Considerémoslas:

"[El reconocimiento del dinero] requiere que cada participante acepte la existencia y la validez del dinero en la creencia de que hay aceptación mutua por parte de los otros" (Searle, Making 58).

"Si hay reconocimiento colectivo de algo como dinero, ese reconocimiento colectivo puede estar constituido por el hecho de que cada persona reconoce el dinero y hay conocimiento mutuo entre los participantes de que todos reconocen el dinero" (Searle, Making 58).

Señalemos primero las características comunes y luego cierta diferencia fina. El punto central que quiere enfatizar Searle es que el reconocimiento colectivo no parece involucrar estados mentales compartidos en un sentido fuerte. Para que la existencia o validez de una institución sea reconocida por un conjunto de individuos, solo es necesario que: (a) cada uno de ellos la reconozca separadamente y (b) cada uno de ellos sepa que los otros también la reconocen. La debilidad de estos criterios se hace patente si los comparamos con los que requiere una acción colectiva bastante más robusta como la cooperación ${ }^{5}$.

Podemos aquí referir a un caso paradigmático de cooperación: caminar juntos (Gilbert, "Acting" 24). Para que dos personas caminen o vayan juntas hacia un sitio no es suficiente que cada una de ellas tenga la intención de caminar o ir hacia ese sitio y que cada una de ellas sepa que la otra está caminando o yendo hacia ese sitio. Para ilustrar el punto: dos personas que caminan en dirección al estadio, luciendo la camiseta del equipo local, no por reconocer que van al mismo lugar caminan juntas; del mismo modo, las personas que toman el mismo avión, y por supuesto saben que lo hacen, no viajan juntas en un sentido que no sea estrictamente espacial.

La diferencia entre las dos definiciones que ofrece Searle reside en la siguiente sutileza. La primera de ellas parece sugerir que parte de que un individuo reconozca una institución depende de que crea que otros la reconocen, como si uno dijera: la reconozco porque otros la reconocen. En la segunda definición esta implicación está ausente. Yo reconozco, por las razones que fuera e independientemente de que

\footnotetext{
${ }^{5}$ Searle mismo (Making 57) concede que el reconocimiento colectivo es una noción más débil que la de cooperación.
} 
otros lo hagan, una institución y además creo que otros la reconocen, por las razones que fuera e independientemente de que yo lo haga. Es evidente que la primera de las definiciones es bastante más colectiva que la segunda, pues de ella se desprende que el reconocimiento de los otros es una condición de cada reconocimiento individual. La segunda definición, por el contrario, arroja la imagen de una sumatoria de reconocimientos individuales aislados y el simple registro por parte de cada uno de esos reconocimientos aislados. Dicho brevemente: según la primera definición, reconocimiento individual y grupal se presuponen mutuamente, emergen juntos; de acuerdo con la segunda, no: el reconocimiento grupal surge del individual, que es autónomo.

Independientemente de la interpretación más o menos exigente que demos de las definiciones, estas se limitan a especificar la forma colectiva del reconocimiento sin decirnos en qué consiste ese reconocimiento mismo: nos ofrecen ciertas distribuciones de estados mentales, pero no nos dicen qué contenido tienen. En este punto crucial, Searle lamentablemente es muy impreciso. Apenas nos indica, acertadamente, que el reconocimiento de una institución no debe identificarse con el respaldo de la misma. Podemos considerar que las tarifas que cobra algún servicio son abusivas. Incluso podemos expresar ese rechazo organizando una movilización. Sin embargo, al momento de pagarlas, las estamos reconociendo. Searle (Making 57) observa que "el reconocimiento o aceptación colectiva... marca un continuo que va desde el respaldo entusiasta hasta el mero ir con la corriente de la estructura".

Podemos aceptar como intuitivamente plausible la concepción de un reconocimiento graduado que va desde el "apoyo entusiasta a la concesión gruñona" (Searle, Making 8). Sin embargo, resulta preciso aclarar algo más de qué se trata ese reconocimiento de una institución del cual solo sabemos, hasta ahora, que no debe identificarse ni con la aceptación ni con el rechazo sino con algo que ambos compartirían. Para hacerlo, conviene retomar la idea del grado mínimo de reconocimiento que Searle caracteriza como dejarse llevar por la estructura. Es de notar que esta expresión sugiere que el reconocimiento se trata de algo mucho más práctico que mental, asunto sobre el cual volveremos más abajo. Por el momento, la forma más apropiada de dar contenido al "dejarse llevar" sin distorsionar el marco intencionalista de Searle es apelar a las funciones de estatus. El reconocimiento colectivo cuenta como reconocimiento de una institución en la medida en que impone estatus a objetos, personas y eventos; estatus que, como se recordará, les 
permiten desempeñar diversas funciones. Podemos, pues, entender el reconocimiento mínimo del que habla Searle, y hasta aquí nos permite avanzar su letra, como una asunción más o menos implícita de que las funciones de estatus se encuentran operando.

\section{Declaraciones}

Parece que, pese a sus insuficiencias, con la idea de reconocimiento colectivo tenemos todos los elementos necesarios para dar cuenta de la existencia y mantenimiento de la realidad social. Mediante el reconocimiento colectivo de ciertos estatus, los objetos y personas conseguirían desempeñar funciones. Las instituciones, a su vez, serían simplemente conjuntos organizados de personas y objetos investidos de estatus funcionales. ¿Qué papel cumple aquí, pues, el lenguaje? ¿Por qué Searle es tan insistente, desde el primer párrafo de su libro (Searle, Making IX), en que la realidad social es una creación lingüística cuando, de su exposición, parece desprenderse que ésta es más bien una creación mental? La respuesta aparece cuando recordamos que la posesión de un estatus se entiende en términos de estar investido de poderes deónticos. De acuerdo con Searle, la realidad institucional es esencialmente normativa. A tal punto que propone el siguiente test: "la manera más simple de establecer si un fenómeno o hecho es genuinamente institucional es preguntarse, ¿su existencia implica poderes deónticos tales como derechos, deberes, obligaciones, requerimientos y autorizaciones?" (Searle, Making 91).

Ahora bien, para explicar este aspecto deóntico o normativo, sostiene Searle, el rol del lenguaje es insoslayable. En la sección siguiente ofreceremos una exposición detallada de esta línea de razonamiento. En lo que resta de esta sección, nos dedicaremos a dar las notas generales de las declaraciones y resaltar algunas conexiones entre estas y el reconocimiento colectivo.

Searle (Making 93) sostiene, en una expresión patente de su orientación intencionalista, que el fundamento de la sociedad está dado por una operación lógica: la creación de una realidad mediante la representación de ésta como existiendo. Esta operación lógica es justamente la función que caracteriza a las Declaraciones, cuyo nombre Searle escribe con mayúscula, y cuya fórmula general es la siguiente: “Nosotros (o yo) hacemos, por Declaración, que sea el caso que la función de estatus Y exista" (Searle, Making 93). 
Para entender qué tienen de especial las Declaraciones, conviene recordar que los actos de habla admiten ser clasificados según su dirección de ajuste. Una aserción o un reporte observacional, actos de habla cognitivos, tienen una dirección de ajuste palabra-mundo, es decir, son los actos de habla los que deben ajustarse a cómo es el mundo. Por otra parte, las órdenes o las expresiones de intención, actos de habla conativos, tienen una dirección de ajuste mundo-palabra, donde los actos de habla se producen para transformar al mundo, buscando que éste se ajuste a ellos. Cuando no hay coincidencia entre lo que se dice y lo que es, si la dirección de ajuste es palabra-mundo, lo que debe cambiar es lo que se dice y si la dirección de ajuste es mundo-palabra, lo que debe cambiar es lo que es. La particularidad de las Declaraciones es que parecen estar dotadas de ambas direcciones de ajuste. En efecto, las declaraciones afirman que algo es el caso, y en ello se parecen a las aserciones, pero mediante esa afirmación, hacen que algo sea el caso, y en ello se parecen a las órdenes o las expresiones de intención. "Cambian el mundo al declarar que un estado de cosas existe y así haciendo que exista ese estado de cosas" (Searle, Making 12). Un caso paradigmático de declaración, observado por John Austin, es el de los bautismos: "Yo nombro esta tierra como la Ciudad de Todos los Santos de la Nueva Rioja", describe el acto de nombrar a la vez que lo realiza, o más precisamente, realiza el acto de nombrar al describirlo.

En cuanto a la relación entre declaraciones y reconocimiento colectivo, Searle nunca acaba de ser claro. A lo largo de su trabajo, se permite frecuentemente afirmaciones como la siguiente: "Todos los hechos institucionales (...) son creados por Declaraciones" (Searle, Making 11) pero también, en otros pasajes, atribuye los mismos poderes al reconocimiento colectivo (Searle, Making 8). De modo que no queda claro aquí sobre cuál de los dos elementos intencionales que invoca descansa el peso fundacional de su argumento. Podemos aventurar, sin embargo, una hipótesis interpretativa respecto a la relación entre reconocimiento y declaraciones. Hipótesis que se apoya en la siguiente cita:

"La afirmación que desarrollaré y defenderé en este libro es que toda la realidad institucional humana es creada y mantenida en existencia por (representaciones que tienen la misma forma lógica que) las Declaraciones FD [de funciones de Estatus]" (énfasis del autor) (Searle, Making 13).

El punto que Searle parece sugerir es el siguiente: las representaciones que crean y mantienen la realidad institucional, representaciones que 
presumimos constituyen el fenómeno del reconocimiento colectivo, se caracterizan por presentar la forma lógica de las declaraciones, a saber, “Nosotros (o yo) hacemos, por Declaración, que sea el caso que la función de estatus Y exista". Si leemos bien al autor, pues, el reconocimiento colectivo acaba por ser el factor decisivo en la institución de la realidad social. Las declaraciones quedarían reducidas a un artefacto que simplemente permite explicitar su forma lógica. No obstante, la insistencia de Searle en la creación de los hechos institucionales por declaración sugiere que está reservando un lugar más relevante para estos actos de habla. Para ver cuál es este lugar, será necesario remitirse, ahora sí, a la conexión entre el lenguaje y poderes deónticos que habíamos pospuesto.

\section{La prioridad del lenguaje}

Searle presenta dos argumentos encadenados a favor de la indispensabilidad del lenguaje y, con ello, a favor de la fundamentación lingüística de la realidad social. El primero busca establecer que sin el lenguaje la creación de poderes deónticos es imposible. El segundo, partiendo de la constatación de que el lenguaje es también un fenómeno normativo en sí mismo, busca mostrar que la normatividad del lenguaje es autónoma y anterior a la normatividad de todos los demás hechos institucionales. Tenemos, pues, el siguiente orden de explicación:

(1) Todos los hechos institucionales, en la medida en que su existencia requiere deontologías, requieren lenguaje.

(2) Las deontologías específicamente lingüísticas son más básicas que las de cualquier otro hecho institucional.

Realidad social, razones y lenguaje

Elaboraremos a continuación el primer argumento (1). Para ello, veamos cómo lo presenta Searle (Making 94-6) a partir de un experimento mental: una población ha construido un muro que resguarda su aldea; con el correr de los siglos, el muro se deteriora hasta convertirse en una simple línea de rocas. A pesar de que físicamente el muro ya no pueda cumplir la función de impedir el paso de los extraños, consigue convertirse, por el peso del uso, en un límite reconocido. El estatus adquirido $Y$, de límite, se entiende en términos deónticos: quien quiera que pretenda cruzarlo está obligado a pedir autorización antes de hacerlo. Searle observa: "Este caso, como cualquier hecho institucional, requiere necesariamente lenguaje o al menos alguna forma de simbolismo" (Making 95). 
Para mostrar por qué las deontologías necesitan del lenguaje para entrar en vigencia conviene observar una característica crucial de su funcionamiento. De acuerdo a Searle (Making 96), la marca distintiva de la presencia de un fenómeno deontológico -obligación, autoridad, permiso, etc.- es que este provee elementos conativos que no son subjetivos, es decir, razones para la acción que son independientes del deseo. Cuando un extranjero se acerca a un limite efectivamente constituido y pide autorización para cruzarlo, actúa según motivos que trascienden sus estados psicológicos individuales, en el siguiente sentido estricto: que pide autorización quiéralo o no. Más aún, esta transcendencia respecto a los estados psicológicos individuales debe entenderse de modo generalizado: no sólo tal o cual extranjero, sino cualquier extranjero debe pedir autorización, quiéralo o no.

Ahora bien, si las razones que caracterizan el funcionamiento de las deontologías son elementos no subjetivos, resulta difícil entender cómo estas podrían surgir a partir de representaciones internas, en principio, inaccesibles a los otros. Se vuelve, pues, necesario, como observa Vicari (190), que las deontologías se expliciten en un medio representacional accesible públicamente. Y este medio es, por supuesto, el lenguaje. Dijimos, en la sección anterior, que el reconocimiento colectivo de que $X$ estaba investido del estatus $\mathrm{Y}$ consistía en que cada miembro del colectivo reconociera ese estatus y además supiera que los otros también lo hacían. Es justamente para cumplir esta segunda condición que el lenguaje se vuelve preciso; la explicitación lingüística es lo que permite coordinar cuáles objetos son reconocidos como teniendo de cuáles estatus. Más aún, una vez puesto a punto este reconocimiento colectivo lingüísticamente mediado, tratar a $X$ como $Y$ ya no es una cuestión del deseo de cada cual, sino una obligación surgida del acuerdo intersubjetivo.

Tenemos, pues, el siguiente esquema fundacional, configurado alrededor de un vínculo, ahora sí, claramente determinado entre declaraciones y reconocimiento colectivo: los hechos institucionales se especifican en términos deontológicos; las deontologías son, efectivamente, instituidas por el reconocimiento colectivo; pero, sin las explicitaciones en un lenguaje público, el reconocimiento quedaría reducido a la mera aceptación privada de esquemas de conducta individuales. Por lo tanto, para que exista un reconocimiento genuinamente colectivo, del cual surgen deontologías independientes de la subjetividad, es preciso que los agentes posean capacidades lingüísticas. De ahí la indispensabilidad del lenguaje. 


\section{La prioridad normativa del lenguaje}

El problema con el esquema fundacional que acabamos de esbozar es que, como es palmario, el lenguaje también constituye también un complejo de deontologías. Por lo tanto, si el funcionamiento de las deontologías requiere lenguaje, nos encontraríamos, tal como reconoce Searle (Making 111), en la primera espiral de un regreso infinito. Para salvaguardar este esquema, Searle (Making 109-115) provee un argumento bastante detallado, que titula ¿Por qué el lenguaje no es una institución social más entre otras?, a favor de la prioridad deontológica o normativa del lenguaje; de acuerdo con este, todas las deontologías necesitan lenguaje, excepto las deontologías del lenguaje. Pasamos a reconstruirlo.

El argumento parte de una constatación, en principio plausible, pero que el grueso del argumento se dedica a cuestionar. Pareciera que el uso del lenguaje admite ser elucidado con el mismo aparato conceptual que se utiliza para elucidar la realidad social. En el lenguaje, distintos eventos materiales, al principio, sonidos $(\mathrm{X})$, cuentan como ciertas oraciones $(\mathrm{Y})$ con un significado determinado. Al igual que la realidad institucional, pues, el lenguaje se encuentra gobernado por reglas constitutivas que, reconocidas colectivamente, invisten objetos o eventos con estatus funcionales.

Searle advierte que, pese a esta similitud superficial, es de fundamental importancia entender que las reglas constitutivas no funcionan del mismo modo para el lenguaje que para el resto de la realidad institucional. De acuerdo al autor, es necesario distinguir dos tipos de poderes deónticos, los semánticos y los extra-semánticos. Los primeros derivan de las reglas constitutivas del lenguaje, que denomina convenciones lingüísticas. Los segundos derivan de convenciones extra-lingüísticas cuya existencia -y aquí descarga el peso fundacional de su argumento- depende de convenciones lingüísticas. Searle apuntala esta diferencia con la siguiente intuición: para que un evento $(X)$ cuente como una oración lingüística $(\mathrm{Y})$, por ejemplo, para que la emisión "el agua es incolora" cuente como una oración que expresa el contenido el agua es incolora, no se necesita más que la convención lingüística que conecta ciertos dibujos o ruidos con contenidos proposicionales. Basta con el significado -con los poderes semánticos creados por las convenciones lingüísticas-para que X cuente como $Y$ en el caso del lenguaje. Por el contrario, por usar un ejemplo de fama, cuando cualquier persona declara marido y mujer a otras dos, no basta simplemente con el significado de la expresión "los declaro marido y mujer" para que el matrimonio se realice; es necesaria la presencia de 
cierta convención extra-lingüística que autoriza solo las oraciones de ciertas personas -digamos, de un funcionario del registro civil- a tener el efecto deseado. Para operar, esta convención extra-lingüística necesita ser explícitamente representada, mientras que las convenciones lingüísticas no. De aquí se desprende la prioridad normativa del lenguaje.

Revisemos un poco más las intuiciones de este argumento. La idea en la que parece apoyarse es que el lenguaje no requeriría que sus normas sean explícitamente representadas porque, de algún modo, su funcionamiento es más simple. Searle a veces pone el punto diciendo que para que en un enunciado cuente, por ejemplo, como una declaración de guerra hace falta una operación ulterior a la simple afirmación "por este acto, declaro la guerra"; en este caso, la designación de una autoridad dotada de ese potestad. Lo que Searle está diciendo, en pocas palabras, es que las declaraciones que crean realidad social guerras o matrimonios- en ciertas circunstancias operan más allá del nivel semántico. Una declaración no es una mera oración significativa. Pero para que ese funcionamiento extra-semántico pueda darse, son necesarias convenciones extra-semánticas que no pueden entrar en fuerza, como hemos mostrado en el argumento previo, si no se representan explícitamente en el lenguaje.

\section{La impotencia de las representaciones}

Expuestas las líneas generales del modelo intencionalista de Searle y sus principales argumentos, pasemos a las consideraciones críticas. En este apartado, queremos presionar los propios razonamientos de Searle hasta concluir que el fundamento de la realidad social no son las representaciones sino la práctica. Procederemos con una táctica de acorralamiento: mostrando cómo el privilegio fundacional que Searle pretende atribuir, primero, al lenguaje y, luego, al reconocimiento colectivo, se va revelando como impotente y desplazando cada vez más, hasta terminar en la práctica.

\section{La impotencia del lenguaje}

Comencemos observando las consecuencias del segundo argumento de Searle. Éste no sólo pretende mostrar que el lenguaje es normativamente más simple que el resto de las instituciones sociales y que, por ello, puede existir independientemente de ellas; quiere convertir esta independencia del lenguaje respecto a los hechos institucionales en una dependencia de los segundos respecto al primero. Este giro puede localizarse fácilmente: 
ocurre cuando Searle pasa de constatar que la institución de hechos sociales requiere más que el significado argumentar que ese elemento extra requerido es una creación lingüística. Queremos cuestionar esta última afirmación y por un motivo simple: lejos de mostrar el poder creador del lenguaje acaba por establecer su impotencia. Desarrollemos. Searle dice que para que la oración "la guerra se ha declarado" pueda efectivamente desencadenar una guerra, debe ser pronunciada por una autoridad competente y que, para que esto ocurra, es preciso que opere la convención extra-semántica que atribuye sólo a esa autoridad, digamos el presidente, la potestad de iniciarla. A su vez, esa condición extra-lingüística, insiste, es creada por el lenguaje. El problema aparece cuando notamos que para la institución de esa convención extra tampoco basta con el significado de las palabras. Si yo digo "sólo el presidente tiene la potestad de declarar la guerra", mi afirmación, sin dejar de tener significado que tiene, no establece esa potestad. Por el contrario, esa oración crea los poderes en cuestión solo en el contexto de convenciones ulteriores, por ejemplo, cuando está incluida en el texto de una Constitución Nacional. La condición extra-lingüística para declarar la guerra, la potestad presidencial, necesita de otra convención extra-lingüística. Y esa otra convención extra-lingüística necesitará, como es patente, una condición extra-lingüística ulterior y así, y así, y así...

Podemos expresar este problema de otro modo. De acuerdo con Searle, la creación de hechos institucionales por medio del lenguaje requiere de convenciones extra-lingüísticas. Pero las convenciones extra-lingüísticas, pese al cambio de nombre, son otros tantos hechos institucionales. La única forma de detener el regreso sería mostrando que ciertos hechos institucionales surgen directamente del uso del lenguaje, sin convenciones extra. Pero esto es precisamente lo que Searle, para apuntalar la diferencia específica del lenguaje y su autonomía, niega. En su intento de mostrar que el lenguaje no es una institución social más, sino la institución social básica necesaria para crear y mantener todas las demás, muestra precisamente que el lenguaje no puede crearlas. Cuando argumenta que sólo el significado de "Declaro que en adelante $\mathrm{X}$ cuenta como $Y^{\prime \prime}$ no basta para instituir una función de estatus, sino que las declaraciones necesitan, para ser efectivas, de condiciones específicas que van más allá del significado, condiciones que a su vez deben ser creadas por Declaración, está simplemente despojando a las declaraciones de todo poder creador. ¿Por qué asume que la primera declaración no alcanza pero la segunda, que crea la convención extra-lingüística, sí? Basándonos en lo que ha dicho, tendríamos que concluir que si la 
primera no alcanza, la segunda tampoco. Lo que hace que el lenguaje sea una institución especial, a saber, que su operación no vaya más allá del significado, es justamente lo que le impide crear las convenciones extrasemánticas que dan cuenta del funcionamiento de las declaraciones.

\section{Del lenguaje al reconocimiento}

La objeción que acabamos de avanzar, si bien aún no consigue, por razones que presentaremos de inmediato, subvertir la fundamentación intencionalista de la sociedad de Searle, arroja una moraleja de importancia: que meramente representarse un estado de cosas, en la medida en que esta es una operación puramente semántica, no alcanza para hacer existir ese estado de cosas. Y es de notar que esta moraleja la hemos recogido del mismísimo argumento de Searle. El significado, por sí mismo, no es suficiente para la institución de nada. A continuación, queremos generalizar esa moraleja para socavar todo el marco intencionalista de Searle.

Si el significado no basta para instituir, por ejemplo, la convención extra-semántica que otorga al presidente la potestad para que sus palabras declaren guerras, ¿de dónde surge esta convención? Retomando la interpretación que hemos ofrecido aquí, podemos decir que esa convención surge del reconocimiento colectivo. Ninguna formulación lingüística instituye nada salvo en el contexto de convenciones colectivamente reconocidas que les otorguen ese poder. Cuando al presidente se le reconoce colectivamente la potestad de declarar la guerra, sus palabras pueden declararla; cuando se reconoce colectivamente el texto de una constitución, sus artículos pueden otorgar potestades presidenciales.

Tenemos, pues, el siguiente esquema revisado: ciertos actos de habla, impotentes por sí mismos, pueden crear realidad social solo en el contexto de convenciones instituidas por el reconocimiento colectivo. Al generalizar esta conclusión, podremos cuestionar la fundamentación intencionalista de Searle. Si el autor antes nos ha mostrado, sensatamente, que el significado de ciertos actos de habla no consigue instituir realidad, ¿por qué deberíamos asumir que los contenidos representacionales de ciertos estados mentales, llamados "reconocimiento colectivo", sí pueden? ¿Cómo ocurre que un puro contenido intencional, que no tiene poderes instituyentes, de repente los adquiera cuando pasa del vehículo del lenguaje al vehículo de la mente? Si proferir "declaro la guerra" no alcanza para declararla, ¿Por qué sí alcanzaría pensar "el presidente puede declarar la guerra" para que el presidente tenga esta potestad? 
Searle, naturalmente, podría reprocharnos que aquí se trata de estados mentales colectivos y no individuales. Lo que hace que el pensamiento "el presidente puede declarar la guerra" invista al presidente de esa potestad es que (a) muchos conciban ese pensamiento y (b) que cada cual sepa que los otros los conciben. Esta respuesta equivale a la siguiente afirmación: las representaciones, lingüísticas o mentales, no son instituyentes solo en virtud de su contenido, pero si ese contenido es concebido por muchos, y además cada cual sabe que los otros los conciben, sí tienen poder para hacer existir estados de cosas sociales. La respuesta, por supuesto, no es muy convincente. ¿Por qué la distribución de un contenido impotente, y la consciencia de esa distribución, de repente hacen que ese contenido no sea más impotente?

\section{Del reconocimiento a la práctica}

Consideramos que si parece convincente decir que el reconocimiento o la aceptación colectivos instituyen hechos sociales, es porque estos términos traen en su sentido ordinario cierto contenido práctico. Es claro que resulta ininteligible decir que alguien reconoce cierta piedra como una deidad, pero en la práctica la trata como a cualquier otro fragmento mineral o que reconoce la vigencia de cierta regla pero la viola constantemente. Esta observación, por elemental que sea, pone de manifiesto un elemento ausente en las definiciones puramente psicológicas del reconocimiento con las que Searle quiere hacer funcionar su sistema, a saber, la crucial importancia de la práctica, de la acción, del comportamiento efectivo para la investidura de las funciones de estatus. El autor habla como si reconocer a $\mathrm{X}$ como teniendo el estatus $\mathrm{Y}$ fuera meramente un asunto de concebir y distribuir ciertos contenidos representacionales, pero es claro que el lugar donde se define la posesión de un estatus es en la práctica (Kukla \& Lance, Yo and Lo 183). Si el reconocimiento o la aceptación colectivas hacen, por ejemplo, de un habitante el Jefe de la aldea, esto es cierto sólo si entendemos esas actitudes colectivas como actitudes prácticas: los aldeanos reconocen a uno de ellos como su jefe cuando se remiten a él en una situación de conflicto interno, le entregan las cosechas para que se encargue de repartirlas, obedecen sus directivas en el caso de una invasión externa, etc. Que un objeto o una persona estén investidos de cierto estatus social es un asunto mucho más práctico que representacional.

De este modo, así como nos vimos antes en la obligación de suplementar las declaraciones con convenciones creadas por el reconocimiento colectivo, ahora nos vemos obligados a suplementar las representaciones 
colectivas con prácticas colectivamente distribuidas. Que ahora sí hemos alcanzado roca fundacional puede verse fácilmente, pues, a diferencia de los contenidos representacionales, la práctica sí tiene poder instituyente propio: que alguien sea el jefe consiste simplemente en que otros lo traten como tal (Brandom, Making 32). Ahora bien, si el reconocimiento es un asunto pragmático, ¿existe alguna forma de salvar el orden de explicación intencionalista? La única opción que queda es insistir en que los aldeanos no tratarían a nadie como su jefe si no pensaran que es el jefe. Mostraremos, para terminar de invertir el sistema intencionalista de Searle, que esta última condición es simplemente irrelevante.

¿Agrega algo suponer que detrás de ciertas prácticas - tratar a X como $Y$ - existen ciertas representaciones explícitas que dicen " $X$ cuenta como $Y^{\prime \prime}$ ? Consideramos que, en la medida en que el problema a elucidar son los fundamentos de la realidad social, esta superestructura mental es prescindible. Para mostrar el punto, consideremos dos opciones. Primero, un caso, por cierto no poco frecuente en la vida social, en que las representaciones que los agentes se hacen de sus instituciones no concuerdan con lo que hacen en la práctica ${ }^{6}$. Un caso como este: oficialmente Jon ha sido designado por el Consejo de los Sabios como el Jefe de la aldea debido a que es el hijo mayor del último Jefe; sin embargo, los éxitos militares de su hermano menor, Ong, le han provisto de popularidad, de un caudal de recursos de los que se ha hecho en sus invasiones y de un ejército que lo idolatra. A todos los efectos prácticos, Ong se encarga de organizar la vida de la aldea: las personas acuden a su consejo, administra los bienes saqueados, ordena que las cosechas tengan como propósito prioritario alimentar las tropas, etc. A pesar de ello, ningún aldeano se atrevería a decir o siquiera pensar que el Jefe es Ong, pues los mecanismos oficialmente reconocidos arrojan que el Jefe es Jon. El punto es simple de ver: cuando la práctica y la representación entran -por una situación enrarecida como la que describimos- en conflicto, lo que manda para establecer quién está investido de poder y quién no es la práctica.

La única forma de salvar la prioridad de las representaciones sería trazando una distinción entre lo que los aldeanos dicen o incluso piensan que reconocen y lo que realmente reconocen (¿quizá inconscientemente?). Así, Searle podría argumentar: aunque ninguno ose explicitarlo ante sus compatriotas, todos los aldeanos en su fuero interno se dicen “Ong es el Jefe" y por ello actúan como actúan. Respecto a esta

\footnotetext{
${ }^{6}$ Muchos estudios empíricos registran casos de este tipo; por mencionar solo un ejemplo clásico, Malinowski (Crimen).
} 
maniobra, podemos decir que se limita a agregar un estrato mentalista de explicación que no hace diferencia alguna. Seamos más claros. La movida que atribuimos a Searle consiste en identificar, detrás de cada actividad A, una representación que diga algo como "Voy a hacer A" que pretendidamente la explicaría. El posible desfasaje entre representación y práctica que observábamos en el párrafo anterior queda conceptualmente bloqueado con esta movida. Pues, hagan lo que hagan los agentes, la representación que se les atribuye es justamente la que dicta hacer lo que efectivamente se hizo. Se trata de una solución a priori, basada en especificar los contenidos de las representaciones a partir de los contenidos de las acciones efectivas.

Respecto a esta solución, notemos dos cosas. En primer lugar, que insistir en la fundamentación intencionalista ya califica, a esta altura, como un capricho. Toda la labor explicativa respecto a la posesión de las funciones de estatus y los poderes deontológicos que acarrean es hecha por la práctica. Es lo que los agentes hacen lo que define qué funciones revisten a cada objeto o persona. Sobreañadir a esto un vínculo explicativo más que se limitaría a repetir "pero nadie haría A si no pensara previamente en hacer $\mathrm{A}^{\prime}$, aun cuando fuera cierto, es simplemente irrelevante. Ocurriría lo mismo si detrás de los estados mentales quisiéramos adivinar la voluntad de algún dios. Diríamos: "pero nadie pensaría en hacer A si un dios no hubiera implantado en ellos este pensamiento". La afirmación podría incluso ser cierta, pero, nuevamente, no sería informativa.

En segundo lugar, observemos que la prioridad, en el orden lógico, de la psicología o la intencionalidad por sobre la práctica es sólo aparente. Pues la maniobra que, por caridad interpretativa, atribuimos a Searle, define los contenidos de las representaciones en función de los contenidos de las acciones. Teóricamente, sólo es posible individuar qué reconocen mentalmente los agentes a partir de lo que reconocen en la práctica. Pero entonces, la prioridad lógica corresponde a la segunda.

\section{La especificidad de nuestro argumento}

Presentada nuestra principal objeción y las conclusiones antiintencionalistas que de ella derivamos, quisiéramos ahora, para acabar de dar un contorno específico a nuestro argumento, situarlo en el marco de otras críticas que se han dirigido contra el programa de Searle. 
En primer lugar, podemos mencionar la reserva, avanzada por Jennifer Hornsby, respecto al carácter genuinamente fundacional de la empresa de Searle. En una recensión crítica de The Construction of Social Reality, Hornsby (430) sugiere que la existencia de colectivos, más que emanar de la intencionalidad colectiva, parece ser una condición de ésta. De acuerdo con la autora, resulta difícil suponer que podamos tener estados mentales de algún modo compartidos sin que haya una comunidad, definida de antemano, con la cual compartirlos. Searle ("Responses" 450) enfrenta esta observación aclarando que, según su punto de vista, hasta que no se comparten ciertos estados mentales, no es inteligible hablar de una comunidad. Sin embargo, el punto de Hornsby no carece de sutileza y no admite una respuesta puramente estipulativa. Aunque la autora no lo diga así, el problema que plantea es delicado: es claro que, en lo que refiere a la existencia de instituciones y poderes deónticos en nuestra comunidad, el hecho de que otras comunidades reconozcan colectivamente otras instituciones es irrelevante $\mathrm{o}$, al menos, indirectamente relevante. Teniendo en cuenta esto, el alcance del reconocimiento colectivo, es decir, quiénes y crucialmente cuántos reconocen tal o cual institución, no puede ser el único criterio para delimitar nuestra comunidad de las otras. Hornsby parece, de hecho, apuntar hacia la intuición contraria: que son los límites de la comunidad o el colectivo los que deben estar dados de antemano para que podamos establecer si una institución efectivamente ha sido reconocida colectivamente. En otras palabras, que debemos haber definido de antemano las actitudes de quiénes son relevantes para contar como sumando al reconocimiento de una institución. Ausente esta definición previa, resulta imposible distinguir una institución que no ha sido reconocida por todo (o la mayoría de) el colectivo de una institución reconocida por un colectivo separado, creado a partir de ese reconocimiento. Cualquier reconocimiento, por marginal que sea, crearía una nueva comunidad. De acuerdo con Hornsby (431), esta dificultad afectaría un elemento general del orden explicativo del programa de Searle: su intento de mostrar cómo la realidad social emerge a partir de átomos más pequeños, a saber, los individuos. La propuesta de Searle, en conclusión, no parecería ser compatible conceptualmente con el individualismo metodológico, pues para funcionar tendría que apelar a entidades sociales de un nivel más alto, como las comunidades o los colectivos.

En segundo lugar, tenemos las reservas respecto a la exhaustividad del modelo intencionalista. Estas pueden expresarse con el eslogan no todo lo social depende del reconocimiento colectivo y provienen de dos direcciones. Por un lado, autores como Wilson (149) han observado que 
ciertos animales no-humanos, como los pájaros, exhiben conductas que podríamos llamar propiamente sociales. Sin embargo, resultaría excesivo atribuirles a estos animales, a diferencia de otros cognitivamente más sofisticados como los chimpancés, la habilidad para representarse objetos como teniendo estatus funcionales. Wilson, pues, concede que la realidad social humana depende del reconocimiento colectivo, pero insiste en que la realidad social es más amplia y admite formas, por así decirlo, más primitivas. Zimmermann, por el contrario, está interesado en mostrar que existen fenómenos sociales humanos que escapan a las determinaciones de la intencionalidad colectiva. Así, considera casos en que ciertos individuos actúan en soledad y sin ninguna voluntad explícita o implícita de compartir sus intenciones con otros, es decir, casos en que las intenciones de los individuos no están dirigidas a nadie; y argumenta que, de todas maneras, puede decirse que estos casos se encuentran socialmente determinados en un sentido muy substantivo. Por ejemplo, cuando un campesino aislado prepara una hoguera de acuerdo a las tradiciones de su región (Zimmermann 136). A partir de estos ejemplos, Zimmermann (137) extiende la determinación social a todos los actos humanos, pues la manera en que concebimos el mundo, las posibilidades de acción que estimamos nos abre y nos cierra, vienen dadas por el lenguaje y los conceptos que recibimos de la cultura en que hemos sido constituidos. En suma, al enfatizar que todo acto humano, aún el más solitario, se enmarca en alguna institución social, Zimmermann impugna la identificación de Searle de la realidad institucional con los fenómenos que necesitan de estados mentales compartidos.

Ninguno de estos cuestionamientos apunta en la dirección del nuestro. Si bien podemos conceder a Hornsby que una definición intencionalista del reconocimiento colectivo sólo parece tener sentido en el marco de una comunidad previamente delimitada, no creemos que esto ponga en riesgo el proyecto ontológico de mostrar cómo la realidad social o los colectivos surgen a partir de los individuos. En este sentido, consideramos que las dificultades del esquema de Searle no son dificultades inherentes a un modelo que parte de los individuos; se derivan, por el contrario, de los conceptos representacionales que el autor ha elegido para construir ese modelo. Aunque no tendremos tiempo de argumentar el punto, consideramos que un modelo que, sin dejar de partir de los individuos, enfatice sus actos y no sus estados mentales, puede superar el problema planteado por Hornsby. Pues en este caso los límites de la comunidad vendrían dados por la articulación objetiva 
de acciones públicas. La diferencia entre un acto que es rechazado por una institución colectivamente reconocida y el mismo acto cuando es aceptado por una institución reconocida por otra comunidad puede trazarse perfectamente en términos prácticos: en el primer caso, sería sancionado y, en el segundo, no. En cuanto a los recelos de Wilson, creemos que pueden ser resueltos terminológicamente, tanto en el modelo de Searle como en la versión que hemos esbozado, en función de cuánto decidamos cargar nuestro concepto de "social": podemos hacerlo tan laxo como para incluir cualquier comportamiento grupal o tan exigente como para excluir toda interacción en la que no se encuentren involucradas deontologías. En todo caso, más que de las etiquetas que se utilicen, la clarificación conceptual dependerá de tener las herramientas suficientes para hacer distinciones entre esos fenómenos a lo largo de un gradiente. Finalmente, ponderar las objeciones de Zimmermann sí requiere maniobras conceptuales y no estipulaciones. Consideramos que los casos que introduce Zimmermann y la caracterización de ellos como fenómenos sociales en un sentido sustantivo es perfectamente sensata. Sin embargo, el foco de su argumento falla. El propósito central del programa intencionalista de Searle no es mostrar que en cada evento donde hay involucrada una institución social debe haber también un episodio de intencionalidad colectiva; por el contrario, su propósito es mostrar que, de no ser por la intencionalidad colectiva, las instituciones sociales nunca hubieran existido. Que esas instituciones creadas por el reconocimiento colectivo acaben por constituir la subjetividad de los individuos es un asunto separado, que merece un tratamiento específico. Dicho en otros términos, la empresa de Searle es ontológica y fundacional, no es una operación de análisis conceptual: Searle no intenta mostrar que las oraciones que utilizan conceptos sociales pueden reformularse, con mayor claridad, como oraciones que utilizan conceptos de representaciones colectivas; por el contrario, quiere mostrar que entre las condiciones de posibilidad de las cosas sociales se encuentran las representaciones colectivas. Las observaciones de Zimmermann a los efectos de que no todos los fenómenos sociales involucran intencionalidad colectiva simplemente se desvían de este objetivo fundacional. Searle pretende haber mostrado los fundamentos representacionales de la realidad social, no haber cubierto todos los casos en que legítimamente se utiliza el predicado "social".

Para resumir: en oposición a Hornsby, nuestro argumento no encuentra mayores dificultades en el proyecto de fundar la realidad social a partir de los individuos, pero, en oposición a Zimmermann, sostiene que las 
dificultades de la versión de Searle de este proyecto no surgen de una falta de exhaustividad conceptual sino de la elección de una ontología inapropiada. De acuerdo con nuestra objeción, Searle no ha identificado correctamente los materiales -los building blocks, por así decirlo- a partir de los cuales se erige la realidad social.

Quien sí ha avanzado una línea de cuestionamiento similar a la nuestra es Tony Lawson, portavoz de la escuela del realismo crítico. En un artículo reciente ("Comparing") en que contrasta su ontología social, y la del Grupo de Cambridge", con la de John Searle, Lawson ("Comparing" 14) señala que su proyecto enfatiza las posiciones sociales, las prácticas colectivas y los poderes deónticos asociados a ellas, mientras que el de Searle insiste en la intencionalidad colectiva. Este contraste, prometedor desde el punto de vista que hemos sugerido aquí, se diluye sin embargo rápidamente. En primer lugar, porque no encontramos en la ontología social de Lawson un argumento a favor de la primacía fundacional de la práctica. Si bien es cierto que Lawson, en el manifiesto A Conception of Social Ontology, comienza un inventario de las entidades sociales que reconoce con una consideración de las prácticas colectivas (Lawson, "A Conception" 34), este comienzo no pretende sugerir una jerarquía ontológica. Por el contrario, siguiendo el hilo conductor de las prácticas colectivas, Lawson busca revelar un conjunto de entidades -como las posiciones y las relaciones sociales, las comunidades, los poderes deónticos y las reglas- que se encuentran holísticamente vinculadas. En segundo lugar, porque Lawson respalda un elemento crucial de la explicación intencionalista, a saber, que los poderes deónticos dependen de un reconocimiento de tipo mentalista; en sus propias palabras: "Ciertamente creo que todas las formas de deontología dependen de formas de representación" (Lawson, "Comparing" 15). En el marco de este respaldo general del programa de Searle, Lawson se permite un pequeño disenso: observa que la existencia de ciertas prácticas colectivas, si bien requiere la creencia de que esas prácticas están operando, no necesita ser explícitamente representada en el lenguaje (Lawson, "Comparing" 16); siendo esta una disidencia dentro del orden de explicación internacionalista que aquí hemos intentado impugnar, no nos pronunciaremos respecto a ella.

\footnotetext{
7 El Cambridge Social Ontology Group es uno de los más activos centros de investigación contemporáneos en ontología social. Conformado por investigadores como Tony Lawson, Margaret Archer, Andrew Collier o Stephen Pratten, se considera a sí mismo heredero de la tradición en ontología social fundada por Roy Bhaskar. El trabajo A Conception of Social Ontology, firmado por Lawson, constituye una suerte de manifiesto donde el grupo expone su programa de investigación.
} 
Para terminar de enmarcar nuestro argumento en la literatura, podemos referir una serie de autores que sí abrazan explícitamente la prioridad de la práctica. Robert Brandom, por ejemplo, ha dedicado un extenso volumen (Making) y una serie de trabajos posteriores dedicados a la semántica de Hegel, entre ellos ("Some Pragmatist" y "A Hegelian"), a mostrar que el vocabulario intencional, mentalista o lingüístico, puede analizarse en términos de un vocabulario pragmático-normativo, que hace referencia a reglas y actos. En este sentido, sus argumentos buscan establecer que los fenómenos normativos son lógicamente anteriores a los fenómenos intencionales. De aquí se desprende que el intento de fundar las deontologías sociales en representaciones colectivas constituiría una inversión de las prioridades lógicas. En la misma línea, Joseph Rouse (How Scientific, "Social Practices"), ha presentado un modelo de la institución y el funcionamiento de las prácticas sociales basada en la forma en que diferentes actos se encuentran articulados entre sí, sin hacer referencia a los estados mentales de los practicantes. La orientación pragmatista de Brandom y Rouse ofrece, sin dudas, el punto de partida conceptual a partir del cual hemos desarrollado nuestro argumento. Sin embargo, ninguno de estos autores está preocupado por elucidar la naturaleza de la realidad social. Por el contrario, ubican sus consideraciones acerca de la prioridad de las prácticas y la normatividad en el marco de discusiones más clásicas respecto a la naturaleza del significado, el conocimiento, la objetividad o la ciencia.

\section{Conclusión}

En este trabajo, nos hemos esforzado por presentar, de la manera más caritativa y clara posible, los principales argumentos que avanza Searle en su fundamentación intencionalista de la sociedad. Hemos mostrado que estos argumentos, debido a sus propios supuestos, tienden a sustentar la prioridad instituyente de la práctica y no de las representaciones. Finalmente, hemos sugerido que, dada esta prioridad de la práctica, es plausible deshacerse por completo de los elementos intencionales a la hora de dar cuenta de los fundamentos de la realidad social. 


\section{REFERENCIAS}

Bratman, Michael. “Shared Intention”. Ethics. Oct. 104 (1). 1993: 97-113. Print. Brandom, Robert. "A hegelian model of legal concept determination: the normative fine structure of the judge's chain novel". Hubbs, Graham y Lind, Douglas (Eds.) Pragmatism, law and language. New York: Routledge. 2014. Print.

. "Some pragmatist themes in Hegel's idealism". Tales of the mighty dead (pp. 210-234). Cambridge, MA: Harvard University Press. 2002. Print.

. Making it explicit: reasoning, representing and discursive commitment. Cambridge, MA: Harvard University Press. 1994. Print.

Chant, Sara, Hindriks, Frank \& Preyer, Gerhard. (eds.) From individual to collective Intentionality: New Essays, Nueva York: Oxford University Press. (2014). Print.

Gilbert, Margaret. "Acting together". Joint commitment: How we make the social world. Nueva York: Oxford University Press. (2014). Print.

Hornsby, Jennifer. (1997). "Collectives and intentionality". Philosophy and Phenomenological Research. Jun. 57 (2). 1997: pp. 429-434. Print.

Kukla, Rebecca \& Lance, Mark. Yo and Lo: The pragmatic topography of the space of reasons. Cambridge, MA: Harvard University Press. 2009. Print.

Lawson, Tony. "Comparing conceptions of social ontology: emergent social entities and/or institutional facts?" Journal for the Theory of Social Behaviour. Dec. 46 (4). 2016: 359-399. Print.

. "A conception of social ontology". Pratten, Stephen (Ed.). Social ontology and modern economics. New York: Routledge. 2015. Print.

Malinowski, Bronislaw. Crimen y costumbre en la sociedad salvaje. Barcelona: Planeta-De Agostini. 1985. Impreso.

Rouse, Joseph. "Social practices and normativity". Philosophy of the Social Sciences. Mar. 37 (1). 2007: pp.1-11. Print.

. How scientific practices matter: reclaiming philosophical naturalism. Chicago: University of Chicago Press. 2002. Print.

Sánchez Cuenca, Ignacio. "A behavioural critique of Searle's theory of institutions". Tsohatzidis, S. (Ed.) Intentional acts and institutional facts: essays on John Searle's social ontology. Dordrecht: Springer. 2007. Print. 
Searle, John, Ferraris, Maurizio \& Condello, Angela. Money, social ontology and law. Nueva York: Routledge. 2019. Print.

. Making the social world: the structure of human civilization. Nueva York: Oxford University Press. 2010. Print.

. "Responses to critics of The Construction of Social Reality". Philosophy and Phenomenological Research. Jun. 57(2). 1997: pp. 449-458. Print. 1995. Print.

The construction of social reality. Nueva York: The Free Press.

Smith, Barry. "John Searle: From speech acts to social reality". Smith, B. (ed.) John Searle. Cambridge, UK: Cambridge University Press. (2003) Print.

Vicari, Giuseppe. "Collective intentionality, language and normativity: a problem and a possible solution for the analysis of cooperation". Epkeina. 5 (1). 2015: 183-207.

Wilson, Robert. "Social reality and institutional facts". Tsohatzidis, S. (Ed.) Intentional acts and institutional facts: essays on John Searle's social ontology. Dordrecht: Springer. 2007. Print.

Zimmermann, Stephan. "Is society built on collective intentions? A response to John Searle". Rivista di estetica. 57. 2014: pp. 121-141. Print. 